\title{
Thermodynamic consistency in dissipative particle dynamics simulations of strongly nonideal liquids and liquid mixtures
}

\section{Citation for published version (APA):}

Trofimov, S. Y., Nies, E. L. F., \& Michels, M. A. J. (2002). Thermodynamic consistency in dissipative particle dynamics simulations of strongly nonideal liquids and liquid mixtures. Journal of Chemical Physics, 117(20), 9383-9394. https://doi.org/10.1063/1.1515774

DOI:

10.1063/1.1515774

Document status and date:

Published: 01/01/2002

\section{Document Version:}

Publisher's PDF, also known as Version of Record (includes final page, issue and volume numbers)

\section{Please check the document version of this publication:}

- A submitted manuscript is the version of the article upon submission and before peer-review. There can be important differences between the submitted version and the official published version of record. People interested in the research are advised to contact the author for the final version of the publication, or visit the $\mathrm{DOI}$ to the publisher's website.

- The final author version and the galley proof are versions of the publication after peer review.

- The final published version features the final layout of the paper including the volume, issue and page numbers.

Link to publication

\section{General rights}

Copyright and moral rights for the publications made accessible in the public portal are retained by the authors and/or other copyright owners and it is a condition of accessing publications that users recognise and abide by the legal requirements associated with these rights.

- Users may download and print one copy of any publication from the public portal for the purpose of private study or research.

- You may not further distribute the material or use it for any profit-making activity or commercial gain

- You may freely distribute the URL identifying the publication in the public portal.

If the publication is distributed under the terms of Article 25fa of the Dutch Copyright Act, indicated by the "Taverne" license above, please follow below link for the End User Agreement:

www.tue.nl/taverne

Take down policy

If you believe that this document breaches copyright please contact us at:

openaccess@tue.nl

providing details and we will investigate your claim. 


\title{
Thermodynamic consistency in dissipative particle dynamics simulations of strongly nonideal liquids and liquid mixtures
}

\author{
S. Y. Trofimov ${ }^{\text {a) }}$ \\ Dutch Polymer Institute, P.O. Box 902, 5600 AX Eindhoven, The Netherlands and \\ Eindhoven University of Technology, P.O. Box 513, 5600 MB Eindhoven, The Netherlands \\ E. L. F. Nies \\ Eindhoven University of Technology, P.O. Box 513, 5600 MB Eindhoven, The Netherlands \\ M. A. J. Michels \\ Dutch Polymer Institute, P.O. Box 902, 5600 AX Eindhoven, The Netherlands and \\ Eindhoven University of Technology, P.O. Box 513, 5600 MB Eindhoven, The Netherlands
}

(Received 4 March 2002; accepted 27 August 2002)

\begin{abstract}
Dissipative particle dynamics (DPD) is a mesoscopic simulation method for studying hydrodynamic behavior of complex fluids. Ideally, a mesoscopic model should correctly represent the thermo- and hydrodynamic properties of a real system beyond certain length and time scales. Traditionally defined DPD quite successfully mimics hydrodynamics, but is not flexible enough to accurately describe the thermodynamics of a real system. The so-called "multibody" DPD (MDPD) is a pragmatic extension of the classical DPD that allows one to prescribe the thermodynamic behavior of a system with only a small performance impact. Here we present a practical improvement to the "multibody" DPD model and test it on a number of single-component examples. We also generalize MDPD to multicomponent systems, which are an important target of DPD studies. The improved model provides a correction for particle correlations in strongly nonideal systems that were neglected in the original MDPD model. The implications of the coarse-graining procedure on the MDPD are discussed. (C) 2002 American Institute of Physics. [DOI: 10.1063/1.1515774]
\end{abstract}

\section{INTRODUCTION}

Mesoscale modeling focuses on predicting the material properties that are determined by the structures and behavior in between the microscopic (atomistic) and macroscopic (continuum) scales. This scale, typically $10-1000 \mathrm{~nm}$ and $1 \mathrm{~ns}-10 \mathrm{~ms}$, is particularly important for understanding polymeric and other complex fluid systems.

While conventional computational methods such as molecular dynamics on the microscopic scale, and fluid dynamics on the macroscopic scale have met with considerable success, the mesoscale of complex fluids still remains beyond their reach. The reason for this state of affairs must be sought in the very high computer-power requirements for the former and the breakdown of the continuum description for the latter. This situation has triggered the development of specifically mesoscopic methods destined to bridge the gap between the micro- and macroscopic approaches.

Dissipative particle dynamics (DPD) is a mesoscopic simulation method for studying hydrodynamic behavior of complex fluids. It was introduced by Hoogerbrugge and Koelman $^{1}$ in 1992 and has since received substantial theoretical support. ${ }^{2,3}$ The method has proven to be useful in the study of the dynamical properties of a wide variety of systems, including colloidal suspensions, ${ }^{4,5}$ dilute polymer solutions, ${ }^{6}$ block-copolymer melts, ${ }^{7,8}$ surfactants, ${ }^{9,10}$ and biological membranes. ${ }^{11,12}$

${ }^{a)}$ Electronic mail: S.Y.Trofimov@tue.nl
Mesoscale modeling usually involves some sort of coarse-graining, i.e., discarding the excessive detail at smaller length and time scales that presumably have no direct influence on the properties of interest. This makes possible the study of larger-scale systems for longer times as compared to purely microscopic approaches like molecular dynamics, which retain (almost) full atomistic detail. It is important, however, that the coarse-grained mesoscopic model correctly reproduces the properties of a real system beyond certain length and time scales, in particular the thermo- and hydrodynamic properties.

DPD as it is traditionally defined quite successfully mimics hydrodynamics, but, unfortunately, is not flexible enough to accurately describe the thermodynamic behavior of a real system. The DPD equation of state (EOS) has a fixed quadratic density dependence irrespective of the parameter values, quite different from the EOS of a real fluid. The aim of this paper is to provide a practical solution to this problem by improving a recent DPD extension, known as the "multibody" DPD (MDPD), introduced by Pagonabarraga and Frenkel. ${ }^{13,14}$

The modification of the "multibody" DPD that we present provides a correction for particle correlations in strongly nonideal systems that were neglected in the original MDPD model; we show how it leads to an accurate thermodynamic description for the Lennard-Jones fluid just above the critical point. We moreover generalize the MDPD conservative-force derivation procedure to multicomponent systems, which are an important target of DPD studies. As an 
illustration we accurately reproduce, using MDPD, the demixing behavior of a binary mixture of compressible Huggins fluids.

The paper is organized as follows: In the next section the DPD method is introduced and the problem of thermodynamic mapping is identified. In Sec. III the "multibody" DPD extension is defined. In Sec. IV the performance of the original MDPD for strongly nonideal systems is evaluated and a practical improvement to the MDPD approach is presented. Also, the implications of the coarse-graining procedure are discussed. In Sec. V a generalization of MDPD to multicomponent systems is presented, and the multicomponent version of MDPD is illustrated with an example.

\section{DPD SIMULATION METHOD}

We first briefly summarize the original DPD simulation method in the (re)formulation by Espanol and Warren, ${ }^{2}$ which has become standard. In DPD the fluid is composed of a number of point (quasi-)particles in continuous space. A particle $i$ is characterized by its position $\mathbf{r}_{i}$, velocity $\mathbf{v}_{i}$, and mass $m_{i}$. For simplicity the masses of all particles are normally chosen to be the same and equal to the reduced unit of mass $m_{i}=m \equiv 1$. The motion of the particles is governed by Newton's equations

$$
\frac{d \mathbf{r}_{i}}{d t}=\mathbf{v}_{i}, \frac{d \mathbf{v}_{i}}{d t}=\mathbf{F}_{i},
$$

with $t$ time and $\mathbf{F}_{i}$ the force acting on particle $i$, due to the presence of other particles. This force is separated into pairwise contributions from conservative, dissipative and random forces,

$$
\mathbf{F}_{i}=\sum_{j \neq i}\left(\mathbf{F}_{i j}^{C}+\mathbf{F}_{i j}^{D}+\mathbf{F}_{i j}^{R}\right) .
$$

All the forces between particles $i$ and $j$ vanish beyond some cutoff radius $r_{c}$, which is usually also chosen as the reduced unit of length, $r_{c} \equiv 1$.

The conservative force determines the thermodynamics of the DPD system, and is defined by the soft repulsion

$$
\mathbf{F}_{i j}^{C}= \begin{cases}a_{i j}\left(1-r_{i j}\right) \hat{\mathbf{r}}_{i j} & \left(r_{i j}<1\right) \\ 0 & \left(r_{i j} \geqslant 1\right),\end{cases}
$$

where $a_{i j}$ is the maximum repulsion strength between particles $i$ and $j$, and $\mathbf{r}_{i j}=\mathbf{r}_{i}-\mathbf{r}_{j}, r_{i j}=\left|\mathbf{r}_{i j}\right|, \hat{\mathbf{r}}_{i j}=\mathbf{r}_{i j} / r_{i j}$. The dissipative and random forces are given by

$$
\begin{aligned}
& \mathbf{F}_{i j}^{D}=-\gamma w^{D}\left(r_{i j}\right)\left(\hat{\mathbf{r}}_{i j} \cdot \mathbf{v}_{i j}\right) \hat{\mathbf{r}}_{i j}, \\
& \mathbf{F}_{i j}^{R}=\sigma w^{R}\left(r_{i j}\right) \theta_{i j} \hat{\mathbf{r}}_{i j},
\end{aligned}
$$

where $\gamma$ is the dissipation strength, $\sigma$ is the noise strength, $w^{D}$ and $w^{R}$ are $r$-dependent weight functions, $\mathbf{v}_{i j}=\mathbf{v}_{i}-\mathbf{v}_{j}$, and $\theta_{i j}$ is a Gaussian white-noise term with the following properties: $\theta_{i j}=\theta_{j i},\left\langle\theta_{i j}(t)\right\rangle=0,\left\langle\theta_{i j}(t) \theta_{k l}\left(t^{\prime}\right)\right\rangle=\left(\delta_{i k} \delta_{j l}\right.$ $\left.+\delta_{i l} \delta_{j k}\right) \delta\left(t-t^{\prime}\right)$.

The choice of the weight functions is not specified by the method, but Espanol and Warren ${ }^{2}$ showed that they should be related according to

$$
w^{D}(r)=\left(w^{R}(r)\right)^{2}
$$

in order for the DPD system to have a well-defined equilibrium state obeying Boltzmann statistics. If Eq. (6) is satisfied, the equilibrium temperature is defined ${ }^{2}$ as $k_{B} T$ $=\sigma^{2} /(2 \gamma)$ (the equilibrium $k_{B} T$ is usually chosen as the reduced unit of energy). For simplicity the weight functions are usually chosen to be similar to the form of the conservative force, so

$$
w^{R}(r)=\sqrt{w^{D}(r)}= \begin{cases}1-r & (r<1) \\ 0 & (r \geqslant 1) .\end{cases}
$$

The dissipative and random forces have two effects: they act as a thermostat, and they allow transport properties such as the viscosity to be tuned without altering the equilibrium thermodynamics. Note that all the forces are pairwise additive, central, and satisfy Newtons Third Law thus conserving linear and angular momentum. The forces depend only on relative positions and velocities making the model Galileaninvariant.

Mapping DPD thermodynamics: It would be beneficial if a mesoscopic model could reproduce correctly the thermodynamic properties of a real system beyond certain length and time scales. This requires the specification of a mapping procedure between the parameters of the model and the physical system it tries to describe. Let us see how it is done for DPD.

The successful use of a mesoscopic simulation method such as DPD always requires some sort of coarse-graining. A number of molecules (or a segment of a polymer chain) of a real fluid is grouped in some way into a "lump" that is represented by a single DPD particle. The obvious result of coarse-graining is that the number density of the coarsegrained particles $\rho$ is different from the number density of the real fluid molecules $\rho^{R}$ (an $R$ superscript marks "real" variables) even if both are expressed in the same units. The ratio $N_{m}=\rho^{R} / \rho$ is the number of real fluid molecules associated with one coarse-grained particle, and is a measure for the level of coarse-graining. Not all of the thermodynamic variables are affected by coarse-graining. The volume of the system is obviously not affected. Moreover, it is known that grouping simple molecules into equally sized nonintersecting clusters leaves the average kinetic energy per degree of freedom unchanged. ${ }^{15,16}$ Therefore, it is reasonable to assume that the temperature is also not changed by a coarse-graining procedure.

The DPD conservative force (3) produces an EOS that can be approximated by the following equation: ${ }^{17}$

$$
p=\rho k_{B} T+\alpha a \rho^{2},
$$

where $\rho$ is the number density of the DPD particles, $a$ is the repulsion strength (for a single-component system the repulsion strength is the same for all particle pairs, $a_{i j}=a$ ), and $\alpha$ is a fitting parameter equal to $0.101 \pm 0.001$ in DPD reduced units (all the numerical values below are given in these units if not stated otherwise). The approximation is very good for $\rho \geqslant 3$ and $a \geqslant 15$. The DPD EOS has rigid quadratic density dependence irrespective of the parameter values. Such an EOS is quite different from the EOS of a real fluid. 
As one can see the thermodynamics of a DPD system is controlled by a single parameter: the repulsion strength $a$. The value of $a$ is usually obtained by matching the compressibility of the DPD model to that of a real system with an incentive to achieve the correct description of the density fluctuations. Groot and Warren ${ }^{17}$ accomplish this by matching the following compressibility-related quantity:

$$
\kappa^{-1}=\left(\rho k_{B} T \kappa_{T}\right)^{-1}=\frac{1}{k_{B} T}\left(\frac{\partial p}{\partial \rho}\right)_{T, N},
$$

where $\kappa_{T}$ is the usual isothermal compressibility,

$$
\kappa_{T}=-\frac{1}{V}\left(\frac{\partial V}{\partial p}\right)_{T, N} .
$$

As one can see, $\kappa^{-1}$ is equal to the reciprocal of the structure factor ${ }^{18}$ of the fluid in the forward scattering direction, $\kappa^{-1}=S^{-1}(0)$, and is a dimensionless property (Groot and Warren call it the dimensionless compressibility). The dimensionlessness is an advantage because it allows avoiding conversion from and to the DPD-specific reduced units. It is not even necessary to establish the values of reduced units in terms of real units. However, one has to keep in mind that $\kappa^{-1}$ scales with the level of coarse-graining, a fact originally overlooked ${ }^{17}$ and taken into account only recently $^{12}$

$$
\kappa^{-1}=N_{m}\left(\kappa^{-1}\right)^{R} .
$$

Calculating $\kappa^{-1}$ from the DPD EOS, and substituting it to this equation we obtain

$$
\kappa^{-1}=1+\frac{2 \alpha a \rho}{k_{B} T}=N_{m}\left(\kappa^{-1}\right)^{R} .
$$

Knowing the value of $\kappa^{-1}$ for a real system (for example for water under standard conditions $\left.\kappa^{-1}=15.9835\right),{ }^{17}$ we are able to find the value of the repulsion strength $a$, ensuring the correct compressibility for a particular value of the DPD particle density $\rho$. In principle, the DPD particle density $\rho$ is a free parameter related to the DPD unit of length: the cutoff radius of the DPD forces. As the CPU time requirements go up with the square of density, it is reasonable to choose the lowest possible density where approximation (8) still holds, i.e., $\rho=3$.

Unfortunately, if one has mapped a particular thermodynamic property of DPD to that of a real system, the other properties are not guaranteed to have the correct values, due to the unphysical quadratic density dependence of the DPD EOS. For example, it is impossible to simultaneously map the isothermal compressibility and the pressure. If we succeed in mapping compressibility, the pressure will have an unrealistically high value, or if we map pressure, the compressibility will be too low. To illustrate this, let us represent the reciprocal of the isothermal compressibility (10) as a function of the pressure of the DPD system (8),

$$
\kappa_{T}^{-1}=\rho k_{B} T+2 \alpha a \rho^{2}=2 p-\rho k_{B} T<2 p .
$$

Clearly, the dimensionless ratio $\kappa_{T}^{-1} / p$ for the DPD system can never exceed the value of 2 , regardless of the values of the parameters used. For water under standard conditions this

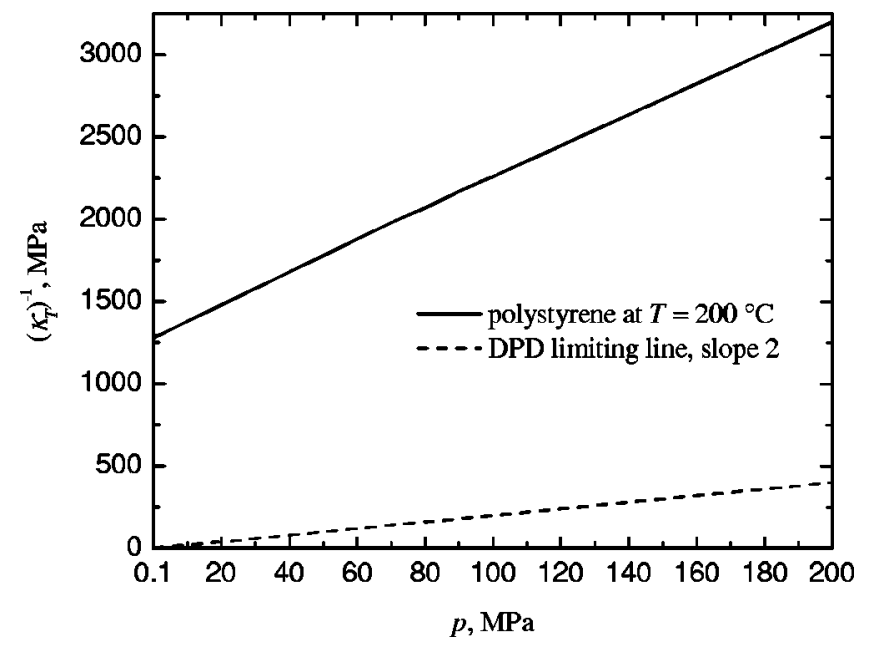

FIG. 1. Reciprocal isothermal compressibility (10) as a function of pressure for polystyrene (solid line, $M=110000, T=200{ }^{\circ} \mathrm{C}$ ) and the limiting line (dashed) with slope 2 for DPD. The lines never cross, meaning that it is impossible to simultaneously map the isothermal compressibility and the pressure for this system.

ratio is equal to $2.22 \times 10^{4}$. For more easily compressible liquids especially at higher pressure this ratio reaches much lower values, however it is still higher than 2. For example, for a typical polymer such as polystyrene $(M=110000$, $T=200{ }^{\circ} \mathrm{C}$ ) at the pressure of $200 \mathrm{MPa}$ it is equal ${ }^{19}$ to 16 (the dependence of $\kappa_{T}^{-1}$ on the pressure for polystyrene and the limiting line with slope 2 for DPD are presented in Fig. 1). Hence, the simultaneous mapping is indeed problematic.

We have to conclude that the traditional DPD is not flexible enough to mimic the thermodynamic behavior of a real system, and an extension seems necessary to overcome this problem. We focus on one possible solution: the "multibody" DPD model introduced by Pagonabarraga and Frenkel. ${ }^{13,14}$

\section{III. "MULTIBODY” DPD MODEL}

The "multibody" DPD model ${ }^{13,14}$ (MDPD) differs from the original DPD in the definition of the conservative forces acting between the DPD particles. Rather than assuming a force (3) that depends only on the interparticle separation, the force is made dependent on the instantaneous local particle density, that in turn depends on positions of all the other neighboring particles. This effectively makes the conservative force in MDPD a many-body force, hence the name of the approach.

The precise form of the local density-dependent conservative forces is chosen based on the desired thermodynamic behavior using the following procedure. We assume that the free energy of an inhomogeneous system with density $\rho(\mathbf{r})$ can be written as

$$
F=\int d \mathbf{r} \rho(\mathbf{r}) \psi(\bar{\rho}(\mathbf{r})),
$$

where $\psi(\rho)$ is the free energy per particle in a homogenous system, and as yet undefined $\bar{\rho}(\mathbf{r})$ is related to the density at (and possibly in the vicinity of) r. Next we rewrite Eq. (14) 
in a discrete form, using the usual $\delta$-function definition for the density, and interchanging the integral and the sum

$$
\begin{aligned}
F & =\int d \mathbf{r}\left\langle\sum_{i} \delta\left(\mathbf{r}-\mathbf{r}_{i}\right)\right\rangle \psi(\bar{\rho}(\mathbf{r})) \\
& =\left\langle\sum_{i} \int d \mathbf{r} \delta\left(\mathbf{r}-\mathbf{r}_{i}\right) \psi(\bar{\rho}(\mathbf{r}))\right\rangle \\
& =\left\langle\sum_{i} \psi\left(\bar{\rho}\left(\mathbf{r}_{i}\right)\right)\right\rangle
\end{aligned}
$$

with the angular brackets $\langle\cdots\rangle$ indicating an NVT-ensemble averaging. The term inside the angular brackets in the last expression can be seen as an instantaneous value of the free energy corresponding to the particle configuration $\left\{\mathbf{r}_{i}\right\}$ (curly brackets denote a set). The term contains the true ensembleaveraged value $\bar{\rho}\left(\mathbf{r}_{i}\right)$, which is not available during a simulation. In order to obtain a free-energy approximation that can be calculated in a simulation, we replace $\bar{\rho}\left(\mathbf{r}_{i}\right)$ with an approximate $\widetilde{\rho}_{i}$ depending on the positions of the particles in the vicinity of particle $i$, and arrive at

$$
\widetilde{F}=\sum_{i} \psi\left(\widetilde{\rho}_{i}\right) .
$$

The instantaneous free-energy approximation $\widetilde{F}$ depends on the positions of all particles, allowing us to derive the effective force as a variation in this energy corresponding to the displacement of a particle. However, as the kinetic motion of the particles automatically accounts for the ideal contribution to the free energy, only the excess part of the free energy should be used to derive the effective interactions,

$$
\mathbf{F}_{i}^{C}=-\frac{\partial \widetilde{F}^{\mathrm{ex}}\left(\left\{\mathbf{r}_{k}\right\}\right)}{\partial \mathbf{r}_{i}}=-\sum_{j} \frac{\partial \psi^{\mathrm{ex}}\left(\widetilde{\rho}_{j}\right)}{\partial \mathbf{r}_{i}} .
$$

Pagonabarraga and Frenkel ${ }^{13,14}$ define the local-density approximation $\widetilde{\rho}_{i}$ as a weighted average of the instantaneous density $\rho\left(\mathbf{r},\left\{\mathbf{r}_{k}\right\}\right)=\Sigma_{j} \delta\left(\mathbf{r}-\mathbf{r}_{j}\right)$ via

$$
\begin{aligned}
\widetilde{\rho}_{i} & =\int d \mathbf{r} w\left(\left|\mathbf{r}-\mathbf{r}_{i}\right|\right) \rho\left(\mathbf{r},\left\{\mathbf{r}_{k}\right\}\right) \\
& =\sum_{j} \int d \mathbf{r} w\left(\left|\mathbf{r}-\mathbf{r}_{i}\right|\right) \delta\left(\mathbf{r}-\mathbf{r}_{j}\right)=\sum_{j} w\left(r_{i j}\right),
\end{aligned}
$$

where $w(r)$ is a weight function vanishing beyond $r_{c}$, and normalized so that $\int_{0}^{\infty} 4 \pi r^{2} w(r) d r=1$. In other words, each particle has a sphere of influence determined by its weight function, and the density approximation at a point is a sum of contributions from nearby particles.

There is a minor inconsistency in the original papers by Pagonabarraga and Frenkel. ${ }^{13,14}$ The authors state that in a homogenous system without particle correlations the density approximation as defined by Eq. (18) gives on average the correct average particle density, $\left\langle\widetilde{\rho}_{i}\right\rangle=N / V=\rho$. However, we have found that this is only true if the contribution of the particle $i$ itself in Eq. (18) is removed. To illustrate this let us calculate the average of $\widetilde{\rho}_{i}$,

$\left\langle\widetilde{\rho}_{i}\right\rangle=\left\langle\frac{1}{N} \sum_{i} \widetilde{\rho}_{i}\right\rangle=\left\langle\frac{1}{N} \sum_{i, j \neq i} w\left(r_{i j}\right)\right\rangle+w(0)$

$$
\begin{aligned}
& =\left\langle\frac{1}{N} \sum_{i, j \neq i} \int d \mathbf{r} \delta\left(\mathbf{r}+\mathbf{r}_{j}-\mathbf{r}_{i}\right) w(|\mathbf{r}|)\right\rangle+w(0) \\
& =\int d \mathbf{r}\left\langle\frac{1}{N} \sum_{i, j \neq i} \delta\left(\mathbf{r}+\mathbf{r}_{j}-\mathbf{r}_{i}\right)\right\rangle w(|\mathbf{r}|)+w(0) \\
& =\rho \int d \mathbf{r} g(\mathbf{r}) w(|\mathbf{r}|)+w(0)=[w g] \rho+w(0),
\end{aligned}
$$

where the square brackets $[\cdots]$ denote the spatial integral over the system volume. Indeed, if $g(r) \simeq 1$ (as assumed by Pagonabarraga and Frenkel $\left.{ }^{13,14}\right)$, then $\left\langle\widetilde{\rho}_{i}\right\rangle \simeq[w] \rho+w(0)$ $=\rho+w(0)$. In order to correct this inconsistency we use the following expression with no self-contribution as the localdensity approximation (denoting it by $n_{i}$ for further reference $)^{20}$

$$
\widetilde{\rho}_{i}=n_{i}=\sum_{j \neq i} w\left(r_{i j}\right) .
$$

Having specified the form of $\widetilde{\rho}_{i}$ we can derive the final expression for the MDPD conservative interactions,

$$
\begin{aligned}
\mathbf{F}_{i}^{C} & =-\sum_{j} \frac{\partial \psi^{\mathrm{ex}}\left(n_{j}\left(\left\{\mathbf{r}_{k}\right\}\right)\right)}{\partial \mathbf{r}_{i}} \\
& =-\sum_{j \neq i}\left(\psi_{\mathrm{ex}}^{\prime}\left(n_{i}\right)+\psi_{\mathrm{ex}}^{\prime}\left(n_{j}\right)\right) w^{\prime}\left(r_{i j}\right) \hat{\mathbf{r}}_{i j} \\
& =\sum_{j \neq i} \mathbf{F}_{i j}^{C},
\end{aligned}
$$

where the primes denote derivatives with respect to the corresponding variables.

Using this formula one can easily calculate the conservative forces acting on the particles for a prescribed free energy. As we can see, although the forces in the MDPD approach are effectively many-body forces, they can be represented as a sum of pairwise components. This makes MDPD not much more computationally demanding than the original DPD method.

The conservative forces (21) depend not only on the free energy, but also on the form of the weight function. However, the weight function is not specified by the method. There are no a priori arguments for the selection of a particular weight function, apart from that it should vanish beyond $r_{c}$ to decrease computational requirements by limiting the number of particle interactions in the spirit of DPD, and be continuous and smooth to avoid spurious jumps in the calculated forces. For simplicity we use a quadratic function related to the weight functions employed in the original DPD (7),

$$
w(r)= \begin{cases}\frac{15}{2 \pi}(1-r)^{2} & (r<1) \\ 0 & (r \geqslant 1) .\end{cases}
$$


The use of this weight function ensures that DPD is recovered as a special case of MDPD. Indeed, if the free energy per particle is set equal to

$$
\psi^{\mathrm{ex}}(n)=\frac{\pi}{30} a n,
$$

the original DPD is recovered exactly. This permits one to fall back on the well-tested DPD method in specific cases, which is useful during the implementation stage.

We have tested the method with another weight function $w_{2}(r)=15 /(4 \pi)\left(2 r^{3}-3 r^{2}+1\right), \quad(r<1) \quad$ having a bellshaped form similar to the weight functions used in the socalled Smoothed Particle Hydrodynamics (SPH) simulation method. ${ }^{21}$ The weight function $w_{2}(r)$ is flat near the origin leading to small repulsive forces for small particle separations. This feature makes the system prone to forming lattice structures with several particles per site at high pressures, a problem common to SPH. The form of the conservative interactions of MDPD bears close resemblance to that of SPH, and when $w_{2}(r)$ is implemented MDPD apparently shares some of its problems. In view of this the weight function $w_{2}(r)$ was rejected.

As a consistency check for the approach let us compare the predictions of the pressure obtained directly from the free energy (thermodynamic route) and that from the derived expression for the forces (21) using the virial route. The pressure derived from the free energy per particle is

$$
p=\rho^{2} \frac{\partial \psi}{\partial \rho}=\rho k_{B} T+\rho^{2} \frac{\partial \psi^{\mathrm{ex}}}{\partial \rho} .
$$

Using the virial route we obtain ${ }^{14}$

$$
\begin{aligned}
p^{\text {virial }} & =\rho k_{B} T+\frac{1}{2 d V}\left\langle\sum_{i, j} \mathbf{r}_{i j} \cdot \mathbf{F}_{i j}^{C}\right\rangle \\
& \simeq \rho k_{B} T+\frac{1}{2 d V} \iint d \mathbf{r} d \mathbf{r}_{1} \rho\left(\mathbf{r}, \mathbf{r}_{1}\right)\left(\mathbf{r}-\mathbf{r}_{1}\right) \cdot \mathbf{F}^{C}\left(\mathbf{r}-\mathbf{r}_{1}\right),
\end{aligned}
$$

where $d$ is the dimensionality of the model, $\rho\left(\mathbf{r}, \mathbf{r}_{1}\right)$ $=\left\langle\sum_{i, j \neq i}^{N} \delta\left(\mathbf{r}-\mathbf{r}_{i}\right) \delta\left(\mathbf{r}_{1}-\mathbf{r}_{j}\right)\right\rangle$ is a two-particle distribution function; we have approximated the discrete sum by an integral and replaced the conservative force between two particles $\mathbf{F}_{i j}^{C}$ from Eq. (21) (depending in general on the positions of all particles) by a continuous version $\mathbf{F}^{C}$ expressed in terms of relative coordinates of these two particles only

$$
\mathbf{F}_{i j}^{C}\left(\left\{\mathbf{r}_{k}\right\}\right) \simeq \mathbf{F}^{C}\left(\mathbf{r}_{i}-\mathbf{r}_{j}\right) .
$$

This is possible if we assume that the density is nearly homogenous, so $\psi_{\mathrm{ex}}^{\prime}(n)$ is most of the time close to its average $\left\langle\psi_{\mathrm{ex}}^{\prime}(n)\right\rangle$

$$
\mathbf{F}^{C}(\mathbf{r})=-2\left\langle\psi_{\mathrm{ex}}^{\prime}(n)\right\rangle w^{\prime}(r) \hat{\mathbf{r}} .
$$

Introducing a new variable $\mathbf{r}_{2}=\mathbf{r}-\mathbf{r}_{1}$, integrating over $\mathbf{r}_{1}$ and using the radial distribution function $g\left(\left|\mathbf{r}-\mathbf{r}_{1}\right|\right)$ $=\rho\left(\mathbf{r}, \mathbf{r}_{1}\right) / \rho^{2}$ we get

$$
\begin{aligned}
p^{\mathrm{virial}} & \simeq \rho k_{B} T+\frac{\rho^{2}}{2 d} \int d \mathbf{r}_{2} g\left(r_{2}\right) \mathbf{r}_{2} \cdot \mathbf{F}^{C}\left(\mathbf{r}_{2}\right) \\
& \simeq \rho k_{B} T+\frac{\rho^{2}}{2 d} \int d \mathbf{r}_{2} g\left(r_{2}\right) \mathbf{r}_{2} \cdot\left(-2\left\langle\psi_{\mathrm{ex}}^{\prime}(n)\right\rangle w^{\prime}\left(r_{2}\right) \hat{\mathbf{r}}_{2}\right) \\
& \simeq \rho k_{B} T+\rho^{2}\left\langle\frac{\partial \psi^{\mathrm{ex}}}{\partial n}\right\rangle\left(-\left[r w^{\prime} g\right] / d\right)
\end{aligned}
$$

where the square brackets $[\cdots]$ denote the spatial integral over the system volume. If there is no local structure in the fluid, that is, $g(r)=1$, then the term $-\left[r w^{\prime} g\right] / d=[w]=1$ and $n_{i}$ is a very good approximation of the density $\rho\{\langle n\rangle$ $=[w g] \rho=\rho$, Eq. (19) $\}$, so that $\left\langle\partial \psi^{\mathrm{ex}} / \partial n\right\rangle=\partial \psi^{\mathrm{ex}} / \partial \rho$. So for ideal (and close to ideal) systems the MDPD pressure coincides with the thermodynamic pressure.

Furthermore, when $\psi^{\mathrm{ex}}(n)$ is linear in $n$ [such as in traditional DPD, Eq. (23)] a good agreement between the thermodynamic and virial pressure is found. In this case $\psi_{\mathrm{ex}}^{\prime}(n)$ is exactly a constant making the quality of the density approximation unimportant. For these conditions it is possible to estimate the error in the MDPD pressure analytically. For example, let us assume that the excess free energy per particle is $\psi^{\mathrm{ex}}(\rho)=A \rho$. According to the procedure presented above we calculate the conservative forces from the given free energy, obtaining

$$
\begin{aligned}
\mathbf{F}_{i j}^{C} & =-\left(\left(A n_{i}\right)^{\prime}+\left(A n_{j}\right)^{\prime}\right) w^{\prime}\left(r_{i j}\right) \hat{\mathbf{r}}_{i j} \\
& =-2 A w^{\prime}\left(r_{i j}\right) \hat{\mathbf{r}}_{i j} \\
& = \begin{cases}\frac{30}{\pi} A\left(1-r_{i j}\right) & \left(r_{i j}<1\right) \\
0 & \left(r_{i j} \geqslant 1\right),\end{cases}
\end{aligned}
$$

which is equivalent to the form of the traditional DPD conservative forces (3) with the repulsion strength equal to $(30 / \pi) A$. According to (8) this leads to the DPD EOS $p^{\text {virial }}=\rho k_{B} T+(30 / \pi) A \alpha \rho^{2}$. However, from thermodynamics the pressure in such a system should be $p=\rho k_{B} T$ $+A \rho^{2}$. So we can see that MDPD underestimates the excess contribution to the pressure by an amount of $1-(30 / \pi) \alpha$ $\simeq 0.036$, which is still an acceptable error. The error is related to $-\left[r w^{\prime} g\right] / d$ not being exactly equal to 1 .

In most other cases of interest, especially for strongly nonideal systems the accuracy of MDPD predictions will suffer. In the next section we check the performance of MDPD on a set of test systems and propose an amendment to the approach of Pagonabarraga and Frenkel that yields a more accurate mapping for strongly nonideal systems.

\section{STRONGLY NONIDEAL SYSTEMS}

We first set out to assess the performance of MDPD for a variety of test systems by trying to mimic their equations of state. We start with simple power law EOS of the form,

$$
p=\rho k_{B} T+A \rho^{k} .
$$

This EOS is artificial and is not relevant for the study of real systems; however, it represents a flexible MDPD testbed. By 
TABLE I. Parameter values used in the MDPD simulation of the system with EOS (33). All values are in DPD reduced units.

\begin{tabular}{ll}
\hline \hline Parameter & Value \\
\hline Box size & $10 \times 10 \times 10$ \\
Noise strength $\sigma$ & 3 \\
Time step & 0.02 \\
Prefactor $A$ & 10 \\
\hline \hline
\end{tabular}

choosing larger values of $k$ stronger nonideal density dependence is obtained and the behavior of the MDPD code can be investigated. Additionally, the information gathered from the tests on the power-law EOSs provides insight on the MDPD behavior for polynomial equations of state. This is useful because a polynomial can quite generically approximate most experimental EOSs.

The case $k=2$ is trivial and has been considered above. Let us go further to steeper EOSs with $k=3$ and $k=4$. We perform a series of simulation runs at particle densities in the range of 2-10 and measure the pressure. To calculate the conservative forces we need the excess free energy per particle $\psi^{\text {ex }}$, corresponding to (33): $\psi^{\mathrm{ex}}(\rho)=A /(k-1) \rho^{k-1}$ [its derivative with respect to the density is $\left.\psi_{\mathrm{ex}}^{\prime}(\rho)=A \rho^{k-2}\right]$.

We use reduced units that are quite standard for DPD. The unit of length is the cutoff radius. The unit of mass is the mass of a particle. The unit of energy is the thermostated temperature of the system multiplied by the Boltzmann constant. The parameters used in the simulation are presented in Table I. The equations of motion are integrated with a DPDspecific self-consistent algorithm ${ }^{24}$ (number of iterations 5).

The obtained EOSs are shown in Figs. 2 and 3 together with the theoretical ones. As we can see the EOS of a MDPD system measured in a simulation has a significant error in pressure; more than $20 \%$ in the case $k=3$, and more that $40 \%$ in the case $k=4$. Let us look at the possible sources of the error. Knowing that for the case $k=2$ the error was mainly related to the term $-\left[r w^{\prime} g\right] / d$ in Eq. (31) being not exactly 1 , we check this by calculating it from the radial

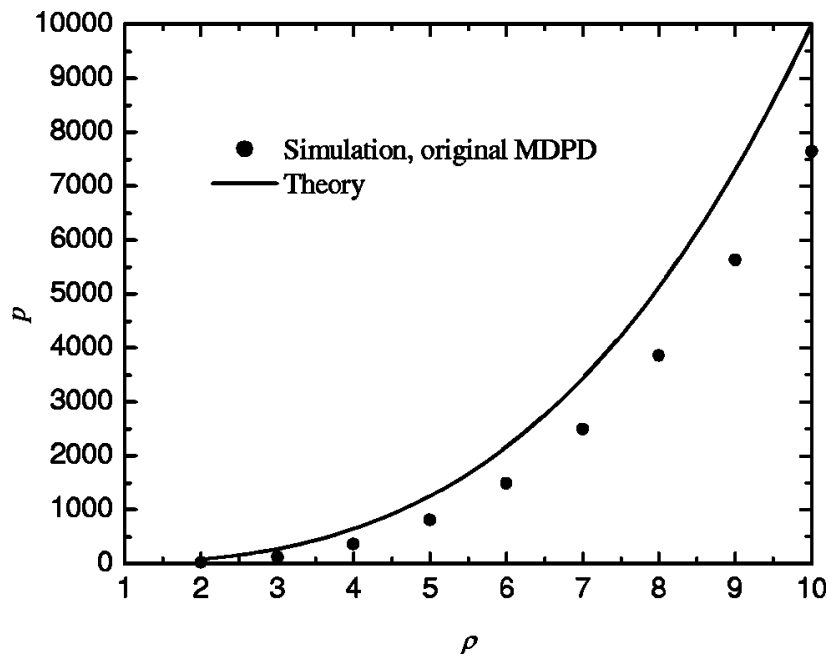

FIG. 2. Pressure as a function of density for the EOS [Eq. (33), $k=3$ ]. Comparison of the simulation results $(\mathbf{)})$, obtained with the original MDPD with the theory (solid line).

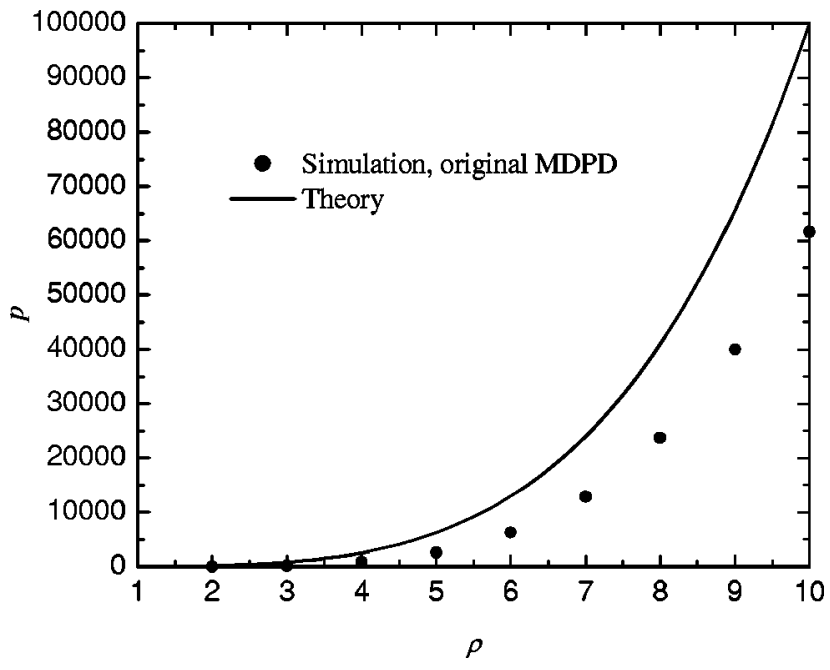

FIG. 3. Pressure as a function of density for the EOS [Eq. (33), $k=4$ ]. Comparison of the simulation results $(\bullet)$, obtained with the original MDPD with the theory (solid line).

distribution function $g(r)$ measured in a simulation. The results are presented in Fig. 4. Except for the point $\rho=2$, the value of $-\left[r w^{\prime} g\right] / d$ is quite close to 1 and is therefore not responsible for the $20 \%-40 \%$ error we observe. Also because it varies slightly with the density and almost in the same way for $k=3$ and $k=4$, it could be easily corrected for (approximately) by multiplying the conservative forces by a constant equal to 1.05 .

The density $\rho=2$ corresponds the situation when average interparticle distances are just slightly smaller than the cutoff radius of the weight function (22). Hence the number of nearby particles sampled by the weight function is very small and thus the quality of the local-density approximation (20) is poor. The quality of the density approximation is very important as it is one of the critical assumptions used in the MDPD conservative-force derivation. So we have to note that MDPD is not likely to be usable at densities $\rho \leqslant 2$.

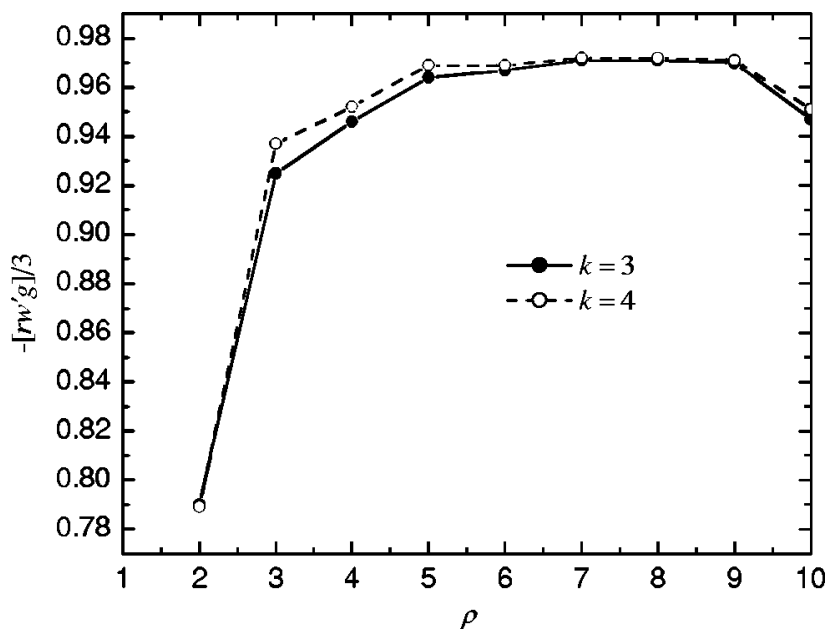

FIG. 4. Simulation results for the term $-\left[r w^{\prime} g\right] / 3$ from Eq. (31) for the system with EOS (33), $k=3$ ( lines are drawn to guide the eye. 


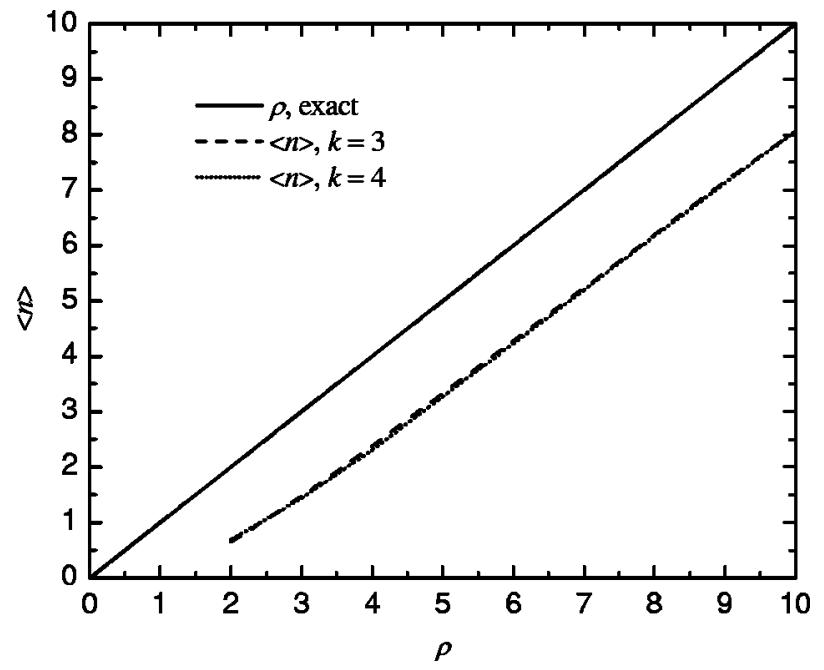

FIG. 5. Simulation results for the average approximated density $\langle\widetilde{\rho}\rangle=\langle n\rangle$, Eq. (20), vs the actual average density $\rho$ for the $\operatorname{EOS~(33),~} k=3$ (dashed line) and $k=4$ (dotted line). The solid line is the relation $\langle n\rangle=\rho$.

To evaluate the quality of the density approximation in the whole density range we plot the measured average approximated density $\langle\widetilde{\rho}\rangle=\langle n\rangle$ versus the actual average density $\rho$ (Fig. 5). It is obvious from the graph that the density is systematically underestimated. This is related to the fact that the strong repulsion forces between particles in the system lead to a correlation hole in $g(r)$. The relative magnitude of the error decreases with increasing density; however, as the CPU-time requirements go up with the square of the density, to make the method practical we have to achieve higher accuracy while remaining in the low-density range $(\rho \leqslant 15)$. We have to stress that this restriction on the DPD particle density $\rho$ is purely method-specific and is related to the choice of a DPD reduced unit of length. It has nothing to do with the density $\rho^{R}$ of a real system simulated.

The approximated local-density curve in Fig. 5 is smooth and almost linear with slope about 1 . It is basically a shift down from the true $\langle\widetilde{\rho}\rangle=\rho$ line. This indicates that the local density $n_{i}$ defined in Eq. (20) provides at least a reasonable starting point to improve on the density approximation. Let us define the local-density approximation $\widetilde{\rho}_{i}$ as a function dependent on $n_{i}$, but not necessarily equal to it. Substituting the new form of $\widetilde{\rho}_{i}$ into Eq. (17) we obtain the corresponding MDPD conservative force expression,

$$
\mathbf{F}_{i}^{C}=-\sum_{j \neq i}\left(\psi_{\mathrm{ex}}^{\prime}\left(\widetilde{\boldsymbol{\rho}}_{i}\right) \widetilde{\rho}^{\prime}\left(n_{i}\right)+\psi_{\mathrm{ex}}^{\prime}\left(\widetilde{\boldsymbol{\rho}}_{j}\right) \widetilde{\rho}^{\prime}\left(n_{j}\right)\right) w^{\prime}\left(r_{i j}\right) \hat{\mathbf{r}}_{i j},
$$

where $\widetilde{\rho}_{k}=\widetilde{\rho}\left(n_{k}\right)$. To determine an appropriate form for $\widetilde{\rho}(n)$ let us first see what conditions it should satisfy. The function must be able to recover approximately the average density value, based on the information about the values of $n_{i}$ calculated during simulation, that is

$$
\left\langle\widetilde{\rho}\left(n_{i}\right)\right\rangle \simeq \rho
$$

for all densities within the region of interest. Assuming that

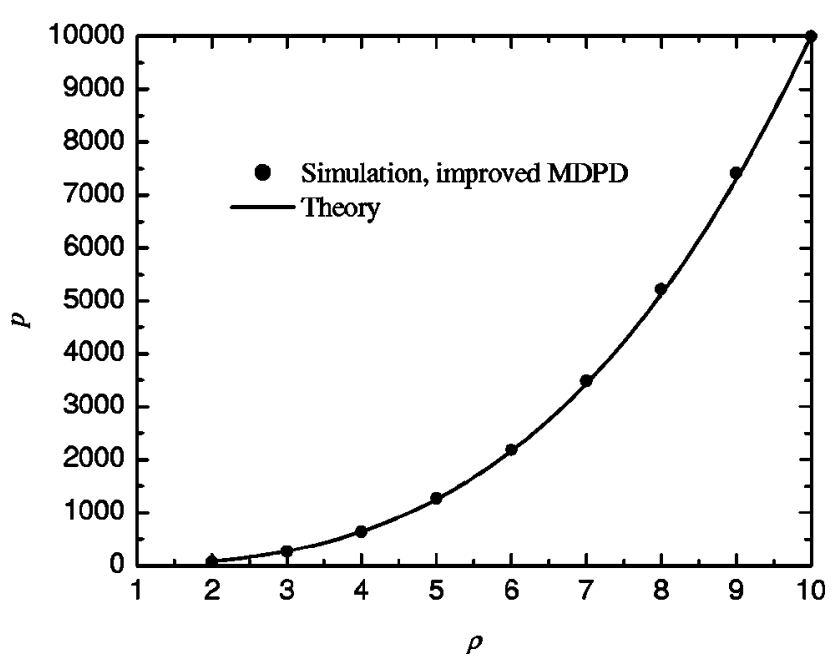

FIG. 6. Pressure as a function of density for the EOS [Eq. (33), $k=3$ ]. Comparison of the simulation results $(\mathbf{)})$, obtained with the improved MDPD with the theory (solid line).

$\widetilde{\rho}(n)$ is almost linear in the region where $n_{i}$ is found most of the time during simulation, we can rewrite condition (35) in the following way:

$$
\widetilde{\rho}(\langle n\rangle) \simeq \rho .
$$

This expression allows us to determine the form of $\widetilde{\rho}(n)$. As $\langle n\rangle$ is in turn dependent on $\widetilde{\rho}(n)$, it must be determined self-consistently. We obtain $\widetilde{\rho}(n)$ using the following iterative procedure. The approximate local density $\widetilde{\rho}(n)$ is represented by a low-order polynomial (in most cases linear). During each iteration $\langle n\rangle(\rho)$ is measured in a simulation and values of parameters in $\widetilde{\rho}(n)$ are obtained by fitting. If the newly measured $\langle n\rangle(\rho)$ substantially disagrees with condition (36) the procedure is repeated.

We use $\widetilde{\rho}(n)=n$ or $\widetilde{\rho}(n)=\rho$ as a first guess. The former is the original MDPD density approximation. The latter is more peculiar: replacing the approximated local density by the known average density. While this substitution leads to an incorrect value for the compressibility, it often allows one to obtain the true $\langle n\rangle$ behavior fast. It is questionable to use this form of local-density approximation in production runs, because the information about the actual average density is not available locally in a simulation, but it works well as a first guess. Simulations with the constant $\widetilde{\rho}(n)=\rho$ are also convenient for initial equilibration of the system. Note, however, that it is necessary to provide some dummy value for $\widetilde{\rho}^{\prime}\left(n_{i}\right)$ as well, usually 1 .

As an illustration of the procedure we determine $\widetilde{\rho}(n)$ corresponding to the EOS (33) with $k=3$ and $A=10$. Using a linear function seems a reasonable choice as the dependence in Fig. 5 is almost linear. We fit this function to the measured data points obtaining $\widetilde{\rho}(n) \simeq 1.047 n+1.54$. After plugging this $\widetilde{\rho}(n)$ into expression (34) we perform a series of simulation runs to measure the EOS of the amended MDPD system. The results presented in Fig. 6 show that a good agreement is obtained after just a single iteration. Ad- 
ditional tests with other EOSs show that the iterative procedure converges very fast and only a single or at most 2 iterations are required. A linear $\widetilde{\rho}(n)$ also proves sufficient for most cases investigated except for the highly nonlinear exponential Tait EOS, ${ }^{25}$ where a quadratic function is needed.

Another way to achieve a more accurate local-density approximation was suggested ${ }^{26}$ for the earlier-mentioned SPH. There a volume $V_{i}=1 / n_{i}$ is associated with each particle. During the simulation the combined volume of all particles $\sum_{i} V_{i}$ is calculated and the densities are rescaled in order for it to be equal to the known total volume of the system $V_{T}$,

$$
\widetilde{\rho}_{i}=n_{i} \frac{\sum_{j} V_{j}}{V_{T}} .
$$

Note, that in this case the density of a given particle depends on the coordinates of all the particles in the system requiring each particle to have the knowledge of the whole system. The authors ${ }^{26}$ argue that this nonlocality breaks the local transport of momentum and therefore the macroscopic hydrodynamic behavior.

\section{A. Implications of coarse-graining}

Let us see what implications coarse-graining has on the force derivation procedure of MDPD. The target information about the thermodynamic behavior of a system we are trying to mimic (for example, in the form of a free-energy expression) is given in terms of noncoarse-grained variables. On the other hand, we need this information expressed in coarsegrained variables to feed it into a MDPD simulation. Hence, we need to specify a general rule for coarse-graining of thermodynamic properties. As discussed above in Sec. II A the volume and the temperature of the system are not affected by coarse-graining and the number of particles scales according to $N=N^{R} / N_{m}$. We suggest the following rule for the coarsegraining of the Helmholtz free energy (and other properties characterizing the system as a whole)

$$
F(N, V, T)=F^{R}\left(N^{R}, V, T\right),
$$

where an $R$ superscript marks real (noncoarse-grained) variables and we assume that all variables are expressed in the same units. Equation (38) leads to similar relations for the free energy per particle and the pressure,

$$
\begin{aligned}
& \psi(\rho, T)=N_{m} \psi^{R}\left(\rho^{R}, T\right), \\
& p(\rho, T)=p^{R}\left(\rho^{R}, T\right) .
\end{aligned}
$$

Let us illustrate this thermodynamics coarse-graining procedure on the simplest system possible: an ideal gas. The free energy of an ideal gas is

$$
\begin{aligned}
& F_{\mathrm{id}}^{R}=N^{R} k_{B} T \ln \left(\rho^{R}\left(\Lambda^{R}\right)^{3} / e\right), \\
& \psi_{\mathrm{id}}^{R}=k_{B} T \ln \left(\rho^{R}\left(\Lambda^{R}\right)^{3} / e\right),
\end{aligned}
$$

where $\Lambda^{R}=\sqrt{2 \pi \hbar^{2} /\left(m^{R} k_{B} T\right)}$ is the thermal wavelength. For use in a simulation we are interested only in the excess part of the DPD free energy per particle $\psi^{\mathrm{ex}}=\psi-\psi^{\text {id }}$. This relation can be rewritten using Eq. (39) as

$$
\begin{aligned}
\psi^{\mathrm{ex}} & =N_{m} \psi^{R}-\psi^{\mathrm{id}} \\
& =N_{m} k_{B} T \ln \left(N_{m} \rho\left(\Lambda^{R}\right)^{3} / e\right)-k_{B} T \ln \left(\rho \Lambda^{3} / e\right) \\
& =k_{B} T\left(N_{m}-1\right) \ln \rho+C(T),
\end{aligned}
$$

where $C(T)$ is independent of density. If we follow the above procedure to derive the MDPD conservative forces based on the free energy (43), we end up with a nonzero force proportional to

$$
\frac{\partial \psi^{\mathrm{ex}}}{\partial \rho}=k_{B} T\left(N_{m}-1\right) / \rho .
$$

These artificial forces can be thought of as responsible for mimicking the internal pressure of fluid elements, which are represented by DPD particles. Note also that if no coarsegraining $\left(N_{m}=1\right)$ is performed these forces disappear, rendering the expected ideal-gas behavior.

Care must be taken when selecting the first guess for $\widetilde{\rho}(n)$ in a simulation with the forces obtained from Eq. (44). Because the density is in the denominator, the error in the density approximation can be magnified tremendously if the absolute value of $\widetilde{\rho}$ is small, and lead to large errors in calculated forces. This may lead to difficulties with initial equilibration and stability of the system. The possible remedy is to fall back to normal DPD during the initial stages of equilibration and in the areas of the system where MDPD is not applicable, for example areas with low particle density.

\section{B. Lennard-Jones fluid EOS}

Combining the results of the previous sections we can now approach a more realistic (yet still a model) system: the Lennard-Jones (LJ) fluid. To apply MDPD we need the expression for the free energy. The exact analytical form of the LJ fluid free energy is unknown. However, approximate but highly accurate EOSs obtained by fitting empirical or semitheoretical equations to the $\mathrm{LJ}$ simulation data, are available in literature. ${ }^{27}$

Fortunately, examination of Eq. (34) shows that to calculate the conservative forces in a simulation one only needs to know the derivative of the excess free energy per particle with respect to the density, and not the free energy itself. Therefore, for a single-component system these forces can be derived from the EOS directly using Eq. (24),

$$
\frac{\partial \psi^{\mathrm{ex}}}{\partial \rho}=p(\rho, T) / \rho^{2}-k_{B} T / \rho .
$$

In fact, the paper $^{27}$ gives the approximate form of the free energy as well, but we follow the simpler EOS route for illustrative purposes, since the free energy in many other cases is not known.

We mimic the LJ EOS isotherm for a temperature just above the critical one $\left(T=1.4, T_{c} \simeq 1.31\right.$ in standard $\mathrm{LJ}$ reduced units) to avoid the vapor-liquid heterogeneous region but still retain the complex form of the curve. As the full empirical LJ EOS is a complex expression with 32 parameters, to simplify the problem we fit a 5-order polynomial to the chosen LJ isotherm and use this polynomial as a target EOS for MDPD, 
TABLE II. Parameter values in DPD reduced units of the 5-order polynomial EOS fitted to the chosen Lennard-Jones isotherm $(T=1.4)$.

\begin{tabular}{cr}
\hline \hline Parameter & \multicolumn{1}{c}{ Value } \\
\hline$\pi_{1}$ & 100.000 \\
$\pi_{2}$ & -33.528 \\
$\pi_{3}$ & 8.234 \\
$\pi_{4}$ & -1.764 \\
$\pi_{5}$ & 0.195 \\
\hline \hline
\end{tabular}

$$
p(\rho, T)=\sum_{i=1}^{5} \pi_{i} \rho^{i} .
$$

The next task is to choose the level of coarse-graining and express the parameter values in DPD units. The level of coarse graining $N_{m}=100$ is set and we map the LJ density of 0.5 to the DPD density of 5 (note the change of the unit of length: the LJ particle density is equal to 0.5 in LJ units, and to $\rho N_{m}=500$ in DPD units) and the LJ reduced temperature of 1.4 to the usual DPD reduced temperature of 1 . This mapping establishes all the DPD reduced units in terms of the LJ reduced units and allows one to express the polynomial target EOS in DPD units. The parameter values in DPD units are presented in Table II.

Using Eq. (45) we derive the MDPD conservative forces from Eq. (46) and then follow the proposed iterative procedure to determine a good local-density approximation. We start with the first guess $\widetilde{\rho}(n)=\rho$ [with dummy $\tilde{\rho}^{\prime}(n)$ $=1.05]$. The value of $\langle n\rangle$ is measured in a set of simulations in the $2-10$ density range. Fitting a linear function to the obtained $\rho(\langle n\rangle)$ data results in $\widetilde{\rho}(n) \simeq 1.062 n+1.39$. After performing another set of simulations with this new $\widetilde{\rho}(n)$, we again observe that a good agreement with the theoretical prediction for the pressure is achieved. The measured EOS is shown in Fig. 7.

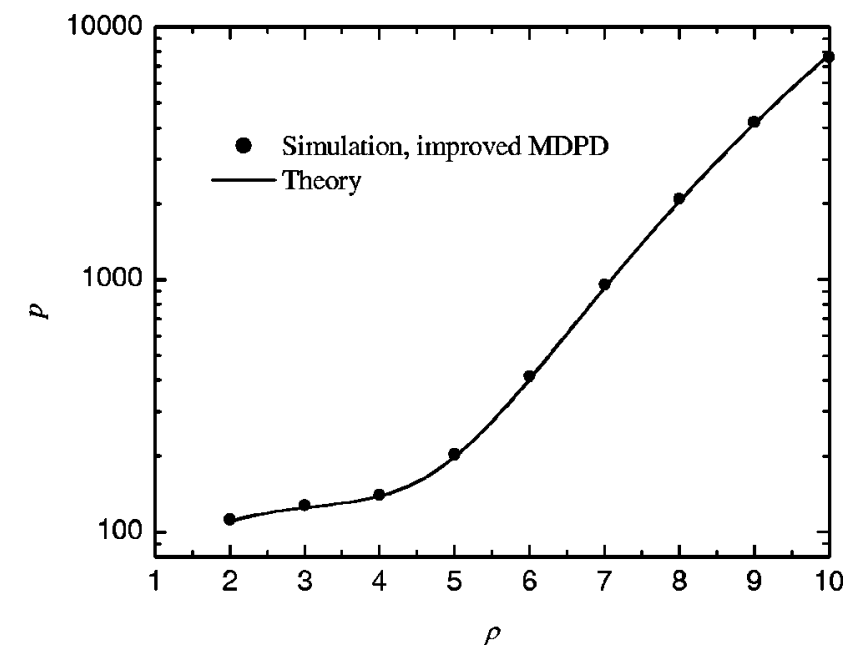

FIG. 7. Pressure as a function of density for the Lennard-Jones EOS. Comparison of the simulation results $(-)$, obtained with the improved MDPD with the theory (solid line).

\section{Making educated first guesses}

Let us look more closely at the simulation with the constant $\tilde{\rho}(n)=\rho$ as the local-density approximation. The constant $\widetilde{\rho}$ leads to $\psi_{\text {ex }}^{\prime}(\widetilde{\rho})$ being constant as well (we denote this constant as $A$ ). This means that a system simulated in such a way has the $\operatorname{EOS}(33), k=2$, and is equivalent to a normal DPD system. However, the constant is different for every density point, $A(\rho)$, so we have a different DPD system at every point. The intended result of these simulations is the measured values of $\langle n\rangle$. It appears that we can obtain the approximate $\langle n\rangle(\rho)$ dependence without additional simulation if we know the relation $\left\langle n_{k=2}\right\rangle(\rho, A)$ for a traditional DPD system,

$$
\langle n\rangle(\rho)=\left\langle n_{k=2}\right\rangle(\rho, A(\rho)) .
$$

This would allow us to skip the first iteration and to determine a density approximation $\widetilde{\rho}(n)$ directly for the cases when a good agreement is normally obtained after a single iteration.

To explore the $\left\langle n_{k=2}\right\rangle(\rho, A)$ landscape we perform a set of simulations for the $3-8$ density range and $A$ from 0 to 100. The simulation results are presented in Fig. 8 . The $\langle n\rangle(\rho)$ dependence corresponding to the Lennard-Jones EOS mimicking system studied in the previous section is also included.

As we can see from Fig. 8(a), $\left\langle n_{k=2}\right\rangle$ is linear in density. As expected $\langle n\rangle$ is equal to the average density value for $A$ $=0$. For $A$ in the region $0-5,\langle n\rangle$ varies quite strongly with $A$, Fig. 8(b). Furthermore, huge fluctuations occur in the instant $n$ value. This makes it difficult to make reasonable predictions of the actual density based on the observed $n$. For $A$ larger than $5,\langle n\rangle$ slowly decreases with $A$ and asymptotically approaches some optimum packing value. Moreover, the fluctuations of $n$ are smaller, so the instant values are most of the time close to the average. In this $A$ range all the $\left\langle n_{k=2}\right\rangle(\rho)$ lines fall very closely to each other. One can see that $A=5$ and $A \gg 1$ form a narrow linear band. Any $\langle n\rangle(\rho)$ curve must lie inside this band. This is the reason why we are able to use similar linear functions to approximate $\langle n\rangle(\rho)$ behavior of different systems. However, care must be taken when using large $A$, as the system becomes very viscous and eventually solidifies. This behavior is unphysical for the real system we are trying to mimic, and therefore the results from such a simulation are useless.

\section{MULTICOMPONENT SYSTEMS}

Though the MDPD method has been applied to binary mixtures, ${ }^{13,14}$ the discussion has been limited to the two special free-energy cases (one of which corresponds to the traditional DPD). No general extension to multicomponent systems of the force derivation procedure has yet been given. However, a detailed description is usually necessary to allow practical application of the method. In the current section we fill this gap and provide details of the extension of MDPD to (coarse-grained) multicomponent systems.

We consider a multicomponent system containing $N_{\alpha}$ particles of component $\alpha$. Greek letters $\alpha, \beta, \gamma$ are used to denote different components and Latin ones- $i, j, k$ - to de- 

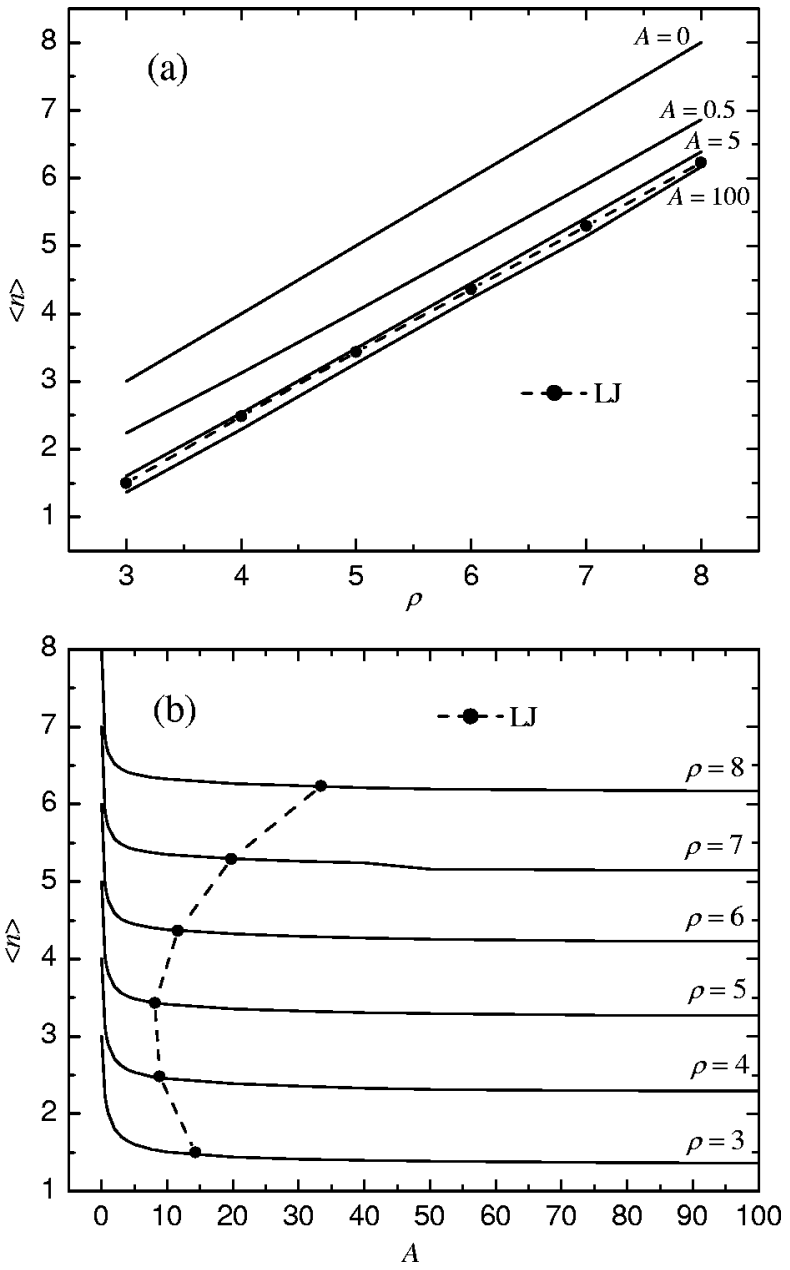

FIG. 8. Simulation results for the average approximate density $\langle n\rangle$ for the system with $\operatorname{EOS~(33),~} k=2$. Such a system is equivalent to the traditional DPD. In (a) the solid lines are the simulation results for $\langle n\rangle$ as a function of the actual average density $\rho$ at the indicated values of the prefactor $A$. In (b) the solid lines are the simulation results for $\langle n\rangle$ as a function of the value of the prefactor $A$ at the indicated values of the actual density $\rho$. () are the results for the Lennard-Jones EOS at the reduced temperature $T=1.4$. The dashed line is drawn to guide the eye.

note individual particles. A term inside curly brackets having a free index denotes a set, for example $\left\{N_{\alpha}\right\}$. Every particle in a system can have neighboring particles of different kinds. To capture this we generalize Eq. (20) and define partial local densities,

$$
\widetilde{\rho}_{i}^{\alpha}=n_{i}^{\alpha}=\sum_{j \in \alpha, j \neq i} w\left(r_{i j}\right) .
$$

The desired free energy has also to be defined as a function of numbers of particles of different components $F\left(\left\{N_{\alpha}\right\}, V, T\right)$. We need to find a MDPD force-derivation procedure analogous to the one in the single component case. Let us represent the excess free energy in the form,

$$
F^{\mathrm{ex}}=\sum_{\alpha} N_{\alpha} \psi_{\alpha}^{\mathrm{ex}}\left(\left\{\rho_{\beta}\right\}\right)
$$

where $\psi_{\alpha}^{\mathrm{ex}}\left(\left\{\rho_{\beta}\right\}\right)$ is an equivalent of the excess free energy per particle for a single-component system. Such a function depends on all the partial densities and represents the inter- action of a particle of type $\alpha$ with its local environment consisting of particles of different types. We can derive the conservative MDPD forces for the free energy (49), obtaining analogously to Eq. (17),

$$
\mathbf{F}_{i j}^{C}=-\left(\frac{\partial \psi_{c(i)}^{\mathrm{ex}}\left(\left\{n_{\alpha}\right\}_{i}\right)}{\partial n_{c(j)}}+\frac{\partial \psi_{c(j)}^{\mathrm{ex}}\left(\left\{n_{\alpha}\right\}_{j}\right)}{\partial n_{c(i)}}\right) w^{\prime}\left(r_{i j}\right) \hat{\mathbf{r}}_{i j},
$$

where $c(i)$ is the component to which particle $i$ belongs $(c(i)=\alpha$, if $i \in \alpha)$, and $\left\{n_{\alpha}\right\}_{i}$ is the set of local densities of different components at the position of particle $i$. As one can see this is a strict generalization of Eq. (21).

Compressible Huggins fluid: Let us demonstrate the multicomponent version of MDPD by attempting to mimic the thermodynamic behavior of a test system: a compressible analog of the Huggins fluid. ${ }^{28}$ Here the model is restricted to monomeric species. The free energy of the compressible Huggins fluid (CHF) has the following form:

$$
\begin{aligned}
F^{R}= & \frac{V}{\nu_{0}} k_{B} T\left(1-\nu_{0} \frac{N^{R}}{V}\right) \ln \left(1-\nu_{0} \frac{N^{R}}{V}\right) \\
& +\sum_{\alpha} N_{\alpha}^{R} k_{B} T \ln \left(\nu_{0} \frac{N_{\alpha}^{R}}{V}\right)-\frac{\nu_{0}}{V} \sum_{\alpha, \beta} N_{\alpha}^{R} N_{\beta}^{R} \varepsilon_{\alpha \beta},
\end{aligned}
$$

where $\nu_{0}$ is the volume of a monomer, $\varepsilon_{\alpha \beta}$ is the absolute value of the interaction energy between particles of types $\alpha$ and $\beta$. The first term in Eq. (51) insures that the system cannot be compressed beyond density $1 / \nu_{0}$. The second term is the combinatorial entropy of mixing the different components. The third term corresponds to interparticle attraction. Equation (51) can be quite easily represented in the form (49) resulting in the following expression for $\psi_{\alpha}$ :

$$
\begin{aligned}
\psi_{\alpha}^{R}= & \frac{k_{B} T}{\nu_{0} \rho^{R}}\left(1-\nu_{0} \rho^{R}\right) \ln \left(1-\nu_{0} \rho^{R}\right)+k_{B} T \ln \left(\nu_{0} \rho_{\alpha}^{R}\right) \\
& -\nu_{0} \sum_{\beta} \varepsilon_{\alpha \beta} \rho_{\beta}^{R} .
\end{aligned}
$$

Following the coarse-graining rule (39) and subtracting the ideal contribution of the DPD system we obtain

$$
\begin{aligned}
\psi_{\alpha}^{\mathrm{ex}}= & \frac{k_{B} T}{\nu_{0} \rho}\left(1-\nu_{0} N_{m} \rho\right) \ln \left(1-\nu_{0} N_{m} \rho\right) \\
& +N_{m} k_{B} T \ln \left(\nu_{0} N_{m} \rho\right) \\
& -N_{m}^{2} \nu_{0} \sum_{\beta} \varepsilon_{\alpha \beta} \rho_{\beta}-k_{B} T \ln \left(\rho_{\alpha}\right)-C(T) .
\end{aligned}
$$

The $\psi$-function derivatives needed for the force computation have the form,

$$
\begin{aligned}
\frac{\partial \psi_{\alpha}^{\mathrm{ex}}}{\partial \rho_{\beta}}= & -\frac{k_{B} T}{\nu_{0} \rho^{2}} \ln \left(1-\nu_{0} N_{m} \rho\right)-N_{m} \frac{k_{B} T}{\rho} \\
& +\left(N_{m}-1\right) \frac{k_{B} T}{\rho_{\alpha}} \delta_{\alpha \beta}-N_{m}^{2} \nu_{0} \varepsilon_{\alpha \beta} .
\end{aligned}
$$

Let us look more closely at the term responsible for mimicking the coarse-grained ideal contribution. As in the case of a single-component system it is only present when 
TABLE III. Parameter values used in the MDPD simulation of the binary mixture of (coarse-grained) compressible Huggins fluids. All values are in DPD reduced units.

\begin{tabular}{ll}
\hline \hline \multicolumn{1}{c}{ Parameter } & Value \\
\hline$\nu_{0}$ & 0.001 \\
$\rho^{R}$ & 800 \\
$x_{A}, x_{B}$ & 0.5 \\
$N_{m}$ & 50 \\
$\rho$ & 16 \\
$k_{B} T$ & 1 \\
Box size & $8 \times 8 \times 16$ \\
Total number of particles & 16384 \\
Noise strength $\sigma$ & 3 \\
Time step & 0.01 \\
\hline \hline
\end{tabular}

coarse-graining is performed $\left(N_{m}>1\right)$. This term produces additional repulsion only acting between particles belonging to the same component and may result in stronger repulsion between particles with more favorable interactions. It turns out that this additional force fulfils both functions of microscopic motion that is lost during coarse-graining; it adds a contribution to the pressure, and it tries to mix the particles of different components. There is also a complication related to the form of the pseudo-ideal contribution. A partial density $\rho_{\alpha}$ is contained in the denominator. If there is a phase separation in the system, $\rho_{\alpha}$ can reach low values, leading to huge forces, which make the simulation unstable. This may require the simulation at higher total density, which is a computationally expensive solution. Another possible remedy, already mentioned above, is to fall back to the original DPD method in such situations. However, one has to keep in mind that this undermines the ability of the system to mimic the desired thermodynamic behavior.

We proceed to test the derived MDPD force expressions in a simulation of a two-component system. In contrast to single-component systems, where we were interested in mimicking the EOS of a system, here we look at mixing properties. The parameters (expressed in DPD units and summarized in Table III) are as follows: $\nu_{0}$ is equal to 0.001 , the total density $\rho^{R}$ is 800 , the average composition $x_{A}=x_{B}=0.5$, the level of coarse-graining $N_{m}$ is the same for the two components and equal to 50. The total DPD particle density $\rho=\rho^{R} / N_{m}=16$. For simplicity a symmetric binary mixture is considered, in which the interactions between particles of the same component are favored $\left(\varepsilon_{A A}=\varepsilon_{B B}\right.$ and $\left.\varepsilon_{A B}=\varepsilon_{A A} / 4\right)$. We set the temperature equal to 1 , and examine the behavior of the system for different effective temperatures by rescaling of the interaction parameters [in this setting the liquid-liquid demixing critical point corresponds to $\left.\varepsilon_{A A}=4 k_{B} T /\left(3 \nu_{0} \rho\right) \simeq 1.67\right]$.

The local-density approximation for a multicomponent system is an extension of the one discussed in Sec. IV. In general for a $m$-component system one can identify $m(m$ $+1) / 2$ "partial" radial distribution functions $g_{\alpha \beta}(r)$ $\left[g_{\alpha \beta}(r)=g_{\beta \alpha}(r)\right]$. Analogously there will be $m^{2}$ local density approximations $\left\langle n_{\alpha \mid \beta}\right\rangle=\left[w g_{\alpha \beta}\right] \rho_{\alpha}$. The term $\left\langle n_{\alpha \mid \beta}\right\rangle$ is the averaged approximated density of particles of type $\alpha$ measured at the position of a particle of type $\beta$. This means that to approximate $m$ partial local densities $\rho_{\alpha}$ we need $m^{2}$

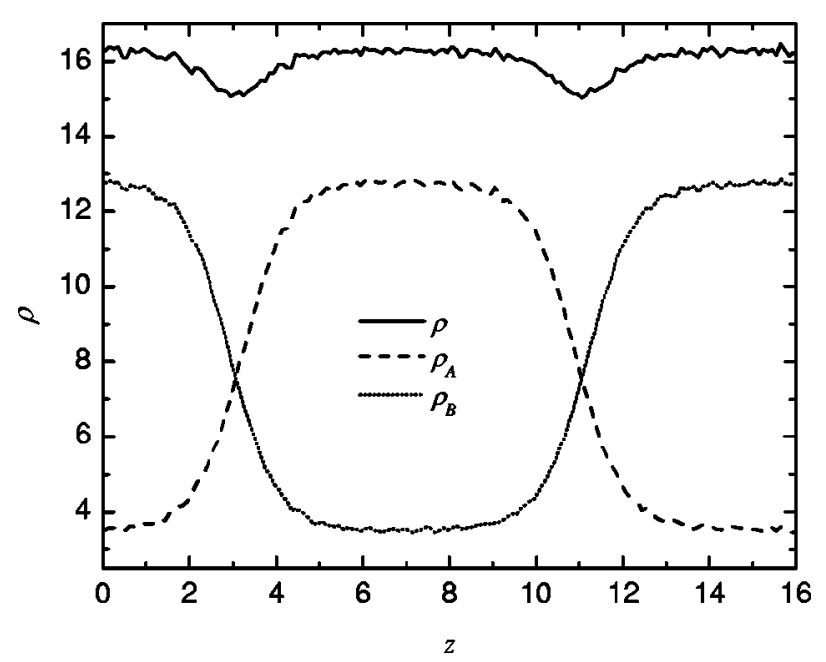

FIG. 9. Averaged density profiles of components A and B, and total density, sampled along the $z$-axis in the MDPD simulation of the CHF system at $\varepsilon_{A A}=1.9$ (solid line: total density, dashed line: density of component A; dotted line: density of component B).

functions $\tilde{\rho}_{\alpha \mid \beta}\left(\left\{n_{\gamma \mid \beta}\right\}\right)$. Consequently in the binary-mixture case we need $2^{2}=4$ functions. Analogously to Eq. (35) we require that $\left\langle\widetilde{\rho}_{\alpha}\right\rangle=\rho_{\alpha}\left(\right.$ for $\left.\varepsilon_{A A}=1.6\right)$ and obtain the following approximation functions:

$$
\tilde{\rho}_{\alpha \mid \beta}=n_{\alpha \mid \beta}+\left\{\begin{array}{cc}
1.57, & \alpha=\beta \\
0.13, & \alpha \neq \beta .
\end{array}\right.
$$

Let us investigate the phase diagram of the system. The system is initially prepared with $\varepsilon_{A A}=1.6$, corresponding to a temperature in the homogenous region slightly above the critical point. In accordance with the prescribed behavior the components mix freely and the value of the pressure (954.5) is quite close to the theoretical one (969.4). The system is then quenched to a temperature in the two-phase region by scaling up the interaction energies, whereupon the phase separation is observed. Six different values of $\varepsilon_{A A}(1.8$, $1.9, \ldots, 2.3)$ were investigated. The periodic-boundary conditions and the elongation of the simulation box in the $z$-direction lead in the end to the formation of the two phase layers perpendicular to the z-axis. When the equilibrium is reached the $z$-density profile is sampled (see Fig. 9) and the bulk compositions of the phases are measured.

The resulting phase diagram is shown in Fig. 10. Again a good agreement with the prescribed behavior is achieved. The larger discrepancy for $\varepsilon_{A A}<1.9$ may be attributed to the fact that the interfaces between phases are very wide and occupy most of the simulation box, hence it is difficult to measure the correct bulk compositions. The discrepancy for $\varepsilon_{A A}>2.2$ is probably related to the fact that one of the partial densities reaches small values (about 2) resulting in a breakdown of the local-density approximation.

\section{CONCLUSIONS}

The "multibody" DPD proposed by Pagonabarraga and Frenkel $^{13,14}$ is a convenient DPD extension allowing one to prescribe the thermodynamic behavior of a system (at constant temperature) with small impact on the simulation per- 


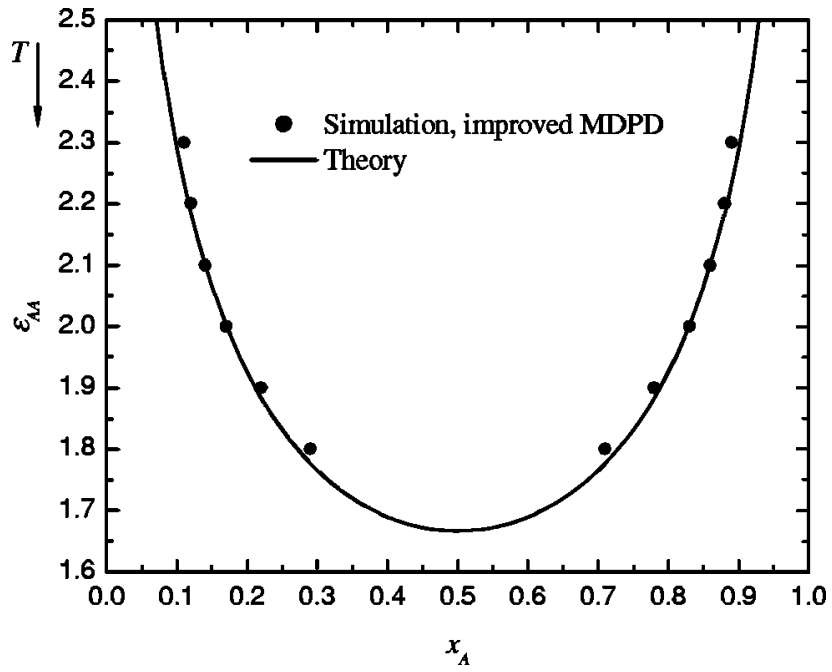

FIG. 10. The phase diagram of the CHF system (, composition of coexisting phases obtained from the MDPD simulation at given values of $\varepsilon_{A A}$; solid line, theoretical binodal).

formance. However, the effect of particle correlations was explicitly neglected in the original MDPD model, making the accuracy of quantitative predictions suffer greatly for strongly nonideal systems, which are of most practical interest. We have repaired this shortcoming of MDPD by correcting for particle correlations in strongly nonideal systems self-consistently using an iterative procedure. By testing our improved MDPD model on a number of examples (including the coarse-grained Lennard-Jones fluid just above the critical point), we have shown that the new model allows one to mimic an arbitrary (realistic) equation of state for a single component system with much better accuracy $(1 \%$ error in pressure) than the original MDPD. Furthermore, we have given a general extension of the MDPD conservative force derivation procedure to multicomponent systems, which is necessary to allow practical application of the method (the discussion had been originally limited to the two special free-energy cases). We demonstrate the multicomponent version of MDPD by accurately reproducing the phase behavior of a (coarse-grained) binary mixture of compressible Huggins fluids.

\section{ACKNOWLEDGMENTS}

We acknowledge I. Pagonabarraga for making a preprint of the paper (Ref. 14) available prior to publication and for answering our numerous questions. We also acknowledge P. van der Schoot for helpful comments.
${ }^{1}$ P. J. Hoogerbrugge and J. M. V. A. Koelman, Europhys. Lett. 19, 155 (1992).

${ }^{2}$ P. Espanol and P. B. Warren, Europhys. Lett. 30, 191 (1995).

${ }^{3}$ C. A. Marsh, G. Backx, and M. H. Ernst, Phys. Rev. E 56, 1676 (1997).

${ }^{4}$ J. M. V. A. Koelman and P. J. Hoogerbrugge, Europhys. Lett. 21, 363 (1993).

${ }^{5}$ E. S. Boek, P. V. Coveney, H. N. W. Lekkerkerker, and P. van der Schoot, Phys. Rev. E 55, 3124 (1997).

${ }^{6}$ A. G. Schlijper, P. J. Hoogerbrugge, and C. W. Manke, J. Rheol. 39, 567 (1995).

${ }^{7}$ R. D. Groot and T. J. Madden, J. Chem. Phys. 108, 8713 (1998).

${ }^{8}$ R. D. Groot, T. J. Madden, and D. J. Tildesley, J. Chem. Phys. 110, 9739 (1999).

${ }^{9}$ S. Jury, P. Bladon, M. Cates, S. Krishna, M. Hagen, N. Ruddock, and P. Warren, Phys. Chem. Chem. Phys. 1, 2051 (1999).

${ }^{10}$ R. D. Groot, Langmuir 16, 7493 (2000).

${ }^{11}$ M. Venturoli and B. Smit, PhysChemComm 2, 45 (1999).

${ }^{12}$ R. D. Groot and K. L. Rabone, Biophys. J. 81, 725 (2001).

${ }^{13}$ I. Pagonabarraga and D. Frenkel, Mol. Simul. 25, 167 (2000).

${ }^{14}$ I. Pagonabarraga and D. Frenkel, J. Chem. Phys. 115, 5015 (2001).

${ }^{15}$ P. Espanol, M. Serrano, and I. Zuniga, Int. J. Mod. Phys. C 8, 899 (1997).

${ }^{16}$ E. G. Flekkoy, P. V. Coveney, and G. de Fabritiis, Phys. Rev. E 62, 2140 (2000).

${ }^{17}$ R. D. Groot and P. B. Warren, J. Chem. Phys. 107, 4423 (1997).

${ }^{18}$ J.-P. Hansen and I. R. McDonald, Theory of Simple Liquids (Academic, London, 1986).

${ }^{19}$ Standard Pressure-Volume-Temperature Data for Polymers, edited by P. Zoller and D. J. Walsh (Technomic, Lancaster, 1995).

${ }^{20}$ The question whether to include the self-contribution into the density approximation (18) has been a controversial one also in the related Smoothed Particle Hydrodynamics method (Ref. 21) (see, for example, Refs. 22 and 23 for the two opposite points of view on this issue). Many authors choose to include the self-contribution, because in practice it may give better results due to partial (and totally uncontrolled) compensation of the error caused by particle correlations. That is a pragmatic solution, only necessary if nothing else is done to improve upon Eq. (18). In this paper we develop a scheme providing a much better local-density approximation accuracy for strongly nonideal systems, hence we choose to exclude the self-contribution for consistency, so that the original MDPD local density approximation is accurate at least under the assumption (of no particle correlations) used by Pagonabarraga and Frenkel (Refs. 13, 14).

${ }^{21}$ O. Kum, W. G. Hoover, and H. A. Posch, Phys. Rev. E 52, 4899 (1995).

${ }^{22}$ O. Flebbe, S. Münzel, H. Herold, H. Riffert, and H. Ruder, Astrophys. J. 431, 754 (1994).

${ }^{23}$ A. P. Whitworth, A. S. Bhattal, J. A. Turner, and S. J. Watkins, Astron. Astrophys. 301, 929 (1995).

${ }^{24}$ I. Pagonabarraga, M. H. J. Hagen, and D. Frenkel, Europhys. Lett. 42, 377 (1998).

${ }^{25}$ C. G. Lyons, J. Mol. Liq. 69, 269 (1996).

${ }^{26} \mathrm{M}$. Serrano and P. Espanol (unpublished).

${ }^{27}$ J. K. Johnson, J. A. Zollweg, and K. E. Gubbins, Mol. Phys. 78, 591 (1993).

${ }^{28}$ P. Cifra, E. Nies, and J. Broersma, Macromolecules 29, 6634 (1996). 